\title{
Structural and Transport Properties of Copper Phthalocyanine (CuPc) Thin Films.
}

\author{
M.M. El-Nahass, F.S.Bahabri *, A.A. AL Ghamdi ** \\ and S.R.Al-Harbi * \\ Faculty of Education, Ain Shams University, Cairo ,Egypt. \\ *Faculty of Girls, Jeddah, Saudi Arabia \\ ** Faculty of science, King Abdul Aziz University, Jeddah, Saudi \\ Arabsia.
}

Thin films of $\mathrm{CuPc}$ of various thicknesses have been deposited onto clean glass substrates using thermal evaporation technique at room temperature. The structural studies, using X-ray diffractometry and IR spectral analysis, showed that the films are mainly of $\beta$ - form with preferential orientation of the plane (100). The dark electrical resistivity and the thermoelectric power measurements were carried out at different temperatures in the range 300-450 K. Room temperature current density-voltage characteristics showed ohmic conduction in the lower voltage range and spacecharge-limited - conductivity $(S C L C)$ in the relatively high voltage. The SCLC are controlled by an exponential distribution of traps above the valence band edge. The temperature dependence of current density allows the calculations of some essential parameters such as the hole mobility, the relative permittivity, the trap concentration, the characteristic temperature and the trap density 


\section{Introduction:}

The phthalocyanines are a class of organic semiconductors which are chemically and thermally stable, and, thus, suitable for the preparation of thin film [1]. They commonly exist in at least two different crystalline phases, the $\alpha$ and $\beta$ - forms [2]. It was reported that the $\beta$ - form is a monoclinic crystal belonging to the $\mathrm{p} 2 / \mathrm{a}$ space group and that there are two phthalocyanine molecules per unit cell. The XRD pattern for the another $\alpha$-form has been reported by several workers [3-7]. A third crystalline modification, called $\gamma$ form, has been also reported [8] which has been proofed to be different from $\alpha$ form ,only in particle size. Another crystal form of phthalocyanine, designated $\chi$ - phthalocyanine, has been also reported [9] The $\beta$ - form is more stable phase and may be thermally derived from the $\alpha$ - form [10]. Phthalocyanines have potential applications in optical logic display devices [11], electrophotography, security printing, gas detectors [12], solar cells [13,14] sensitisers and color filters [13]. However, conductivity of these materials depends on the gaseous environment surrounding the material $[15,16,17]$. The electrical properties of phthalocyanines have been widely studied in the form of sandwich structures, and space-charge -limited conductivity was observed with the current density, showing a power -law dependence on applied voltage [18] .The p-type semiconductivity is, thus, strongly modified by gaseous adsorption and is also dependent on impurities [19] and on film structure [20]. In the present work, the structural properties of $\mathrm{CuPc}$ thin films was studied. The electrical transport properties such as the dark electrical resistivity and the thermoelectric power as well as the space charge limited current are also investigated .

\section{Expermintal Details:}

The $\mathrm{CuPc}$ powder used in this work was obtained from Kodak, England. Thin films of $\mathrm{CuPc}$ have been prepared by conventional thermal evaporation technique, deposited onto pre-cleaned glass substrates kept at ambient conditions using a coating unit (Edwards type E 306 A, England). The evaporator was a quartz crucible source heated by a tungsten coil in a vacuum of $10^{-4} \mathrm{~Pa}$ during deposition. The deposition rate was controlled at $2.5 \mathrm{nms}^{-1}$ using a quartz crystal thickness monitor (Model FTM6, Edwards Co., England). Thickness was determined accurately after deposition using a multiple beam interferometer (NiFE, JUNGNER, 68294). Annealing of the prepared films was carried out at different temperatures in the range (373-523K) for two hours.

The structural properties of $\mathrm{CuPc}$ in powder and in thin films were investigated using $\mathrm{X}$-ray diffraction patterns and infrared spectral techniques. A Philips X-ray diffractometer (model X Pert), with utilized monochromatic Cuk ${ }_{\alpha}$ radiation was used. A continuous scanning technique was employed to observe 
the preferred orientation of the deposited $\mathrm{CuPc}$ crystallite. An accurate determination of the peak intensities was obtained by employing line profile analysis, using a step increment of $0.02^{\circ}$ and an exposure of $1 \mathrm{~s}$.

Infrared spectroscopy on $\mathrm{CuPc}$ films was performed at room teperature using ATI Mattson (Infinity series FTIR) infrared spectrophotometer in the spectral range $4000-400 \mathrm{~cm}^{-1}$. For this study $1 \mathrm{mg}$ of CuPc powder was mixed with $50 \mathrm{mg}$ of vacuum dried IR - grad $\mathrm{KBr}$, as well as thin films of thickness $300 \mathrm{~nm}$ were deposited onto $\mathrm{KBr}$ optically flat substrate.

The dark electrical resistivity $\rho$ of $\mathrm{CuPc}$ thin films was measured by the two probe method in a reasonable temperature range using highly internal impedence electrometer (Keithely $617 \mathrm{~A}$ ) and the temperature was measured using cromel alumel thermocouple and the contacts were made by evaporating high purity $\mathrm{Au}$ electrodes. The thermoelectric power, was measured using the differential- technique[21]. Current-voltage measurments were made using a keithley 617 programable elecrometer with internal power supply .

\section{Results and discussion}

\subsection{Structural investigations}

An X-ray diffraction traces derived from powder $\mathrm{CuPc}$ at $300 \mathrm{~K}$ is shown in Fig. (1.a). Lattice spacings $d_{\mathrm{hkl}}$ were calculated using Bragg's equation together with the Miller indices and the calculated values of $d_{\mathrm{hkl}}$ are compared with the corresponding values given for $\beta$-phase with monoclinic structure in the ICDD Card no. 11-0893 of $\beta$-CuPc. Consequently, the lattice parameters are taken as : $a=1.468 \mathrm{~nm}, \mathrm{~b}=0.498 \mathrm{~nm}, \mathrm{c}=1.960 \mathrm{~nm}$ and $\beta=121.5^{\circ}$.

The obtained crystalline structure of $\mathrm{CuPc}$ thin films, Fig.(1.b), indicates the configuration of $\beta$-form with the single (100) plane as preferred orientation,. Our obtained results differs from that obtained by James et al. [22] using thermal evaporation. The as deposited films were assigned as $\alpha$-phase and converted to $\beta$-phase by heat treatment at $573 \mathrm{~K}$. This may attributed to the modified source of evaporation used in the present. A slow scan for the (100) plane reflection was carried out and the mean crystallite size was estimated from Scherrer's Equation [23] .

$$
L=\frac{k_{s} \lambda}{\beta_{o} \cos \theta}
$$




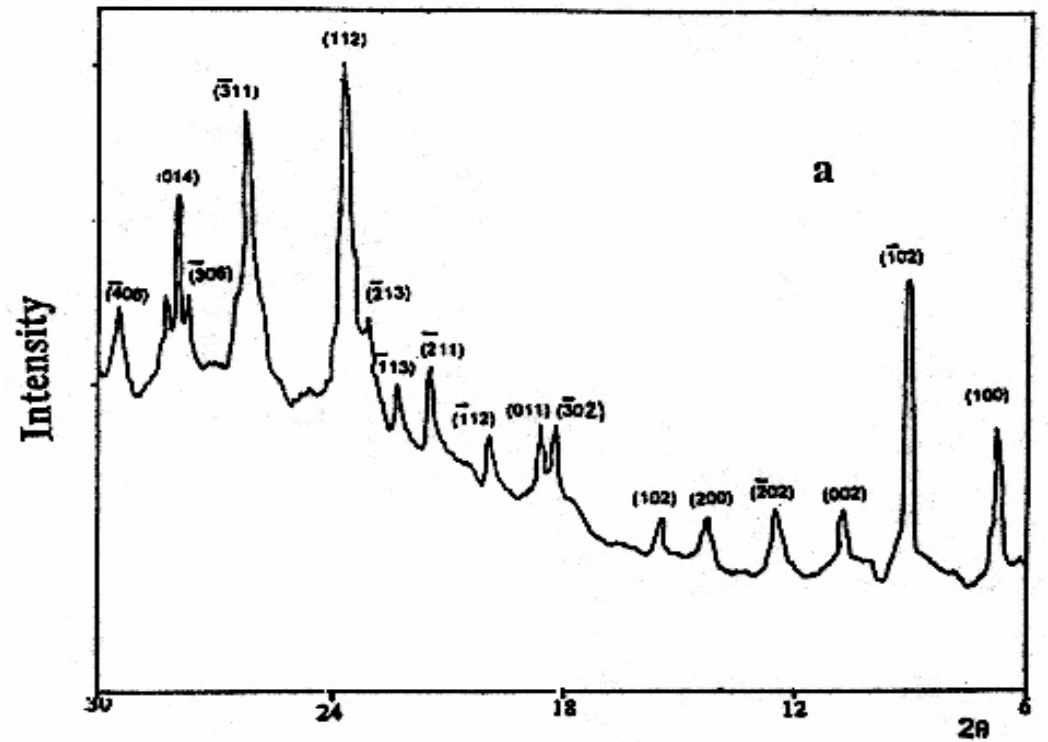

Fig .(1.a): $\mathrm{X}$ - ray diffractometer traces of $\mathrm{CuPc}$ in powder forms.



Fig (1.b) : X - ray diffractometer traces of different $\mathrm{CuPc}$ film thicknesses

where $\lambda$ is the $\mathrm{X}$-ray wavelength $(0.154 \mathrm{~nm}), \beta_{\mathrm{o}}$ the width at half maximum intensity in radians, $\theta$ Bragg's angle and $\mathrm{k}_{\mathrm{s}}$ the Scherrer's constant $=0.9$ [23]. The mean crystallite size, L, represented as a function of film thickness and annealing temperature is depicted in Fig. (2 and 3) respectively. The results show an increase in grain size with increasing film thickness due to the columnar growth with increasing either film thickness or annealing temperatures. The effect of annealing on the crystallite size is shown in 
Fig.(2.b) for a film of $362 \mathrm{~nm}$ thick. As observed, at annealing temperatures $\geq 150^{\circ} \mathrm{C}$, the grain size, L increases markedly with annealing temperature.

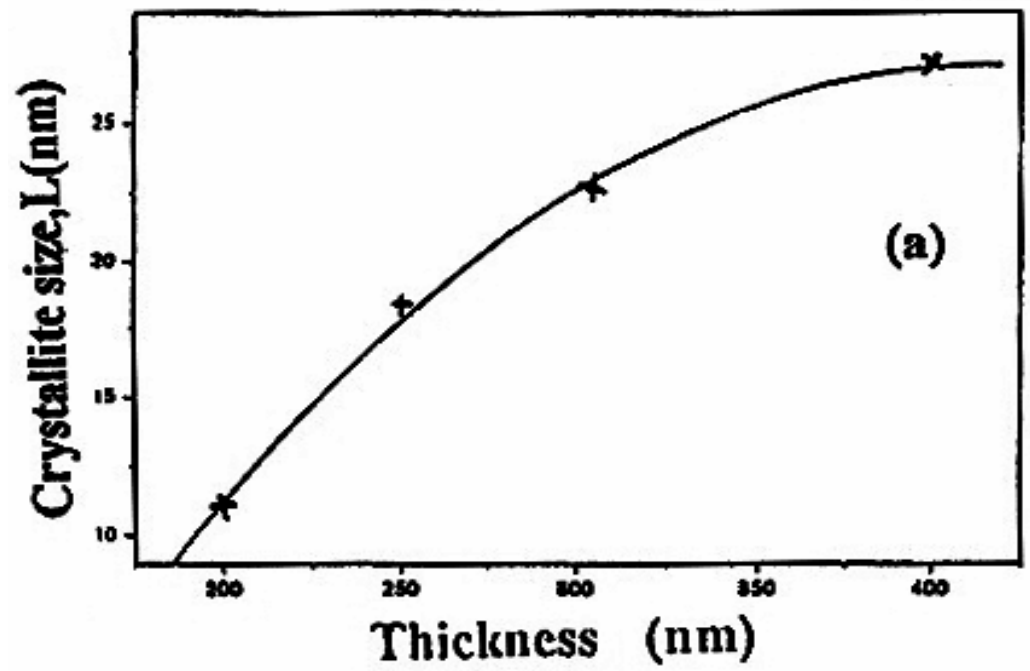

Fig .(2.a): The mean crystallite size $\mathrm{L}$ as a function of film thickness.

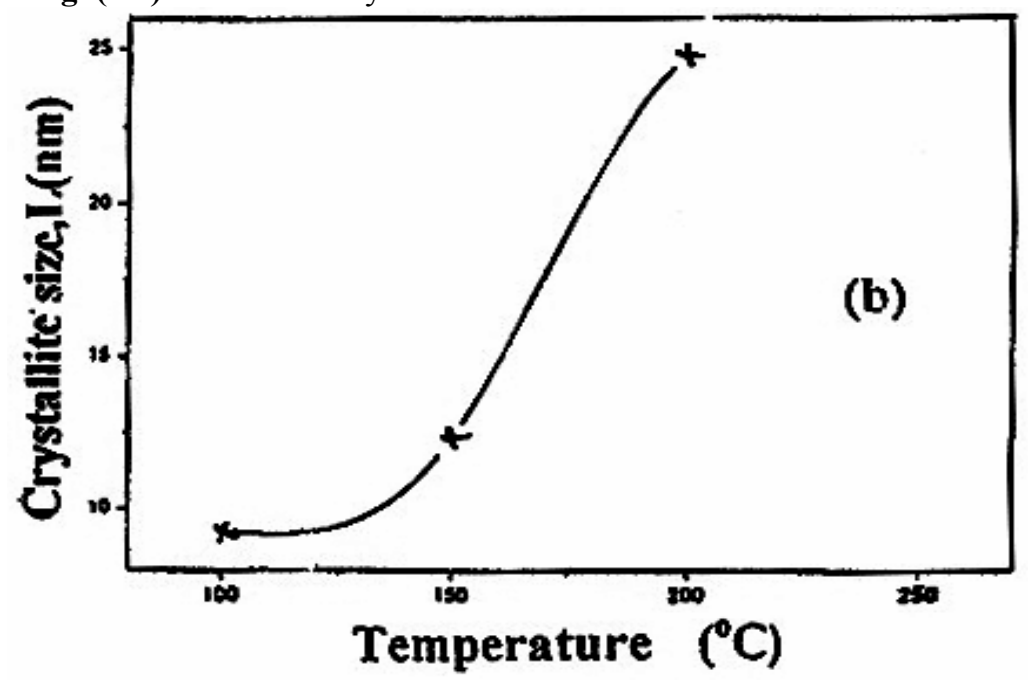

Fig (2.b) : The grain size $\mathrm{L}$ as a function of annealing temperature.

The IR absorption technique may also be used to identify the crystalline nature for both powder and thin films of CuPc. The IR spectra of a substance is markedly dependent on the chemical composition, as well as on its crystalline form [23]. Therefore, and to confirm the results revealed by XRD, the IR spectra of both powder form and thin films are obtained and shown in Fig. ( 3 a, b) at $\left(\cong 733 \mathrm{~cm}^{-1}\right)$ absorption peak existing in both patterns indicate the existence of $\mathrm{CuPc} \beta$-phase structure. 


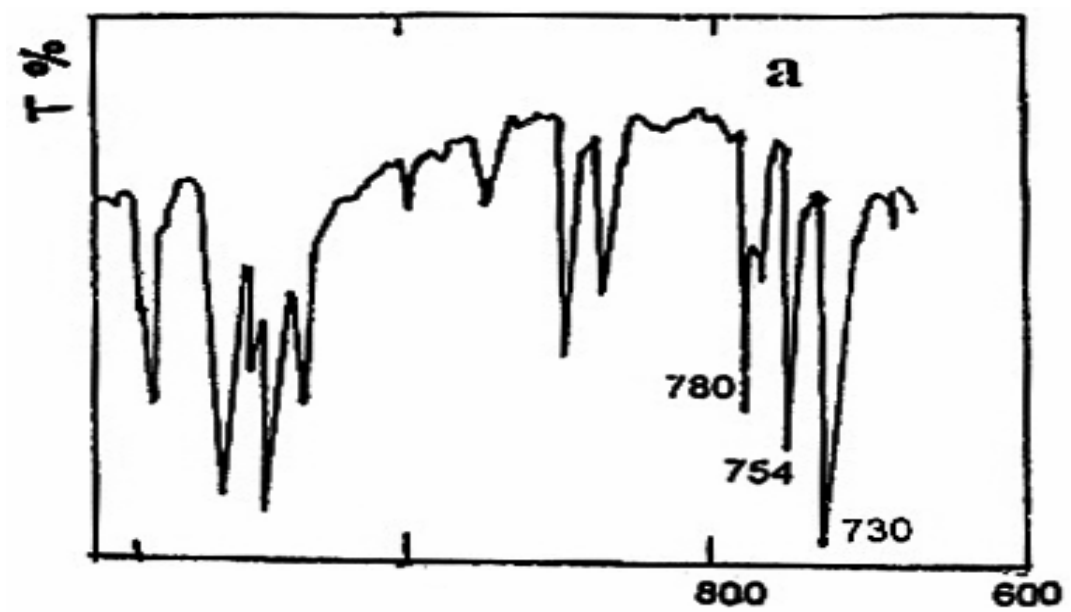

Fig .(3.a): Infrared absorption spectra of $\mathrm{CuPc}$ in powder form.

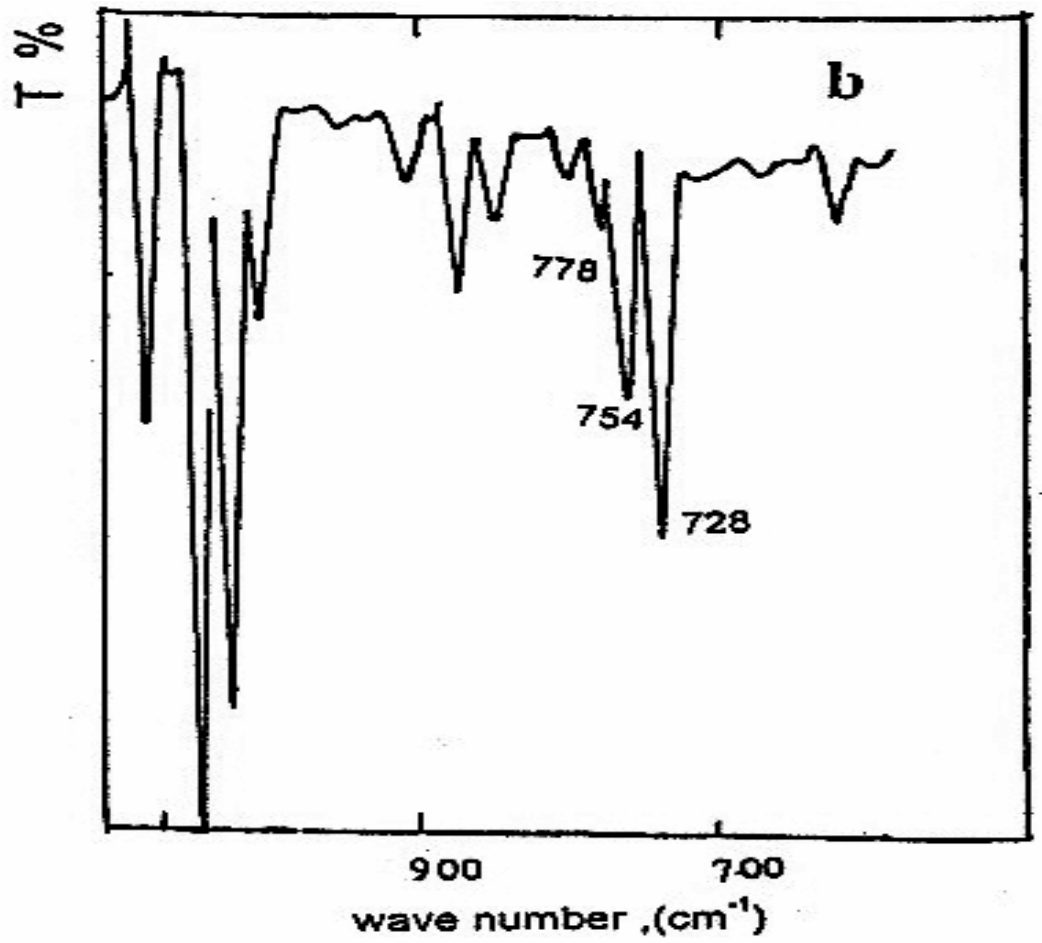

Fig (3.b) : Infrared absorption spectra of $\mathrm{CuPc}$ in thin film form.

\subsection{Dark Electrical resistivity and Thermoelectric power measurements}

The dark electrical resistivity for the as deposited and annealed $\mathrm{CuPc}$ films of different thicknesses is represented in Fig. (4.a) . 
The observed dark resistivity decreases with increasing the film thickness as shown in Fig. (4.a), The same behavior was also reported for both inorganic[24] and organic[25] semiconducting films. This behaviour is attributed to the increase of the CuPc crystallite size with increasing the film thickness [25], as obtained above, Fig. (2.a).

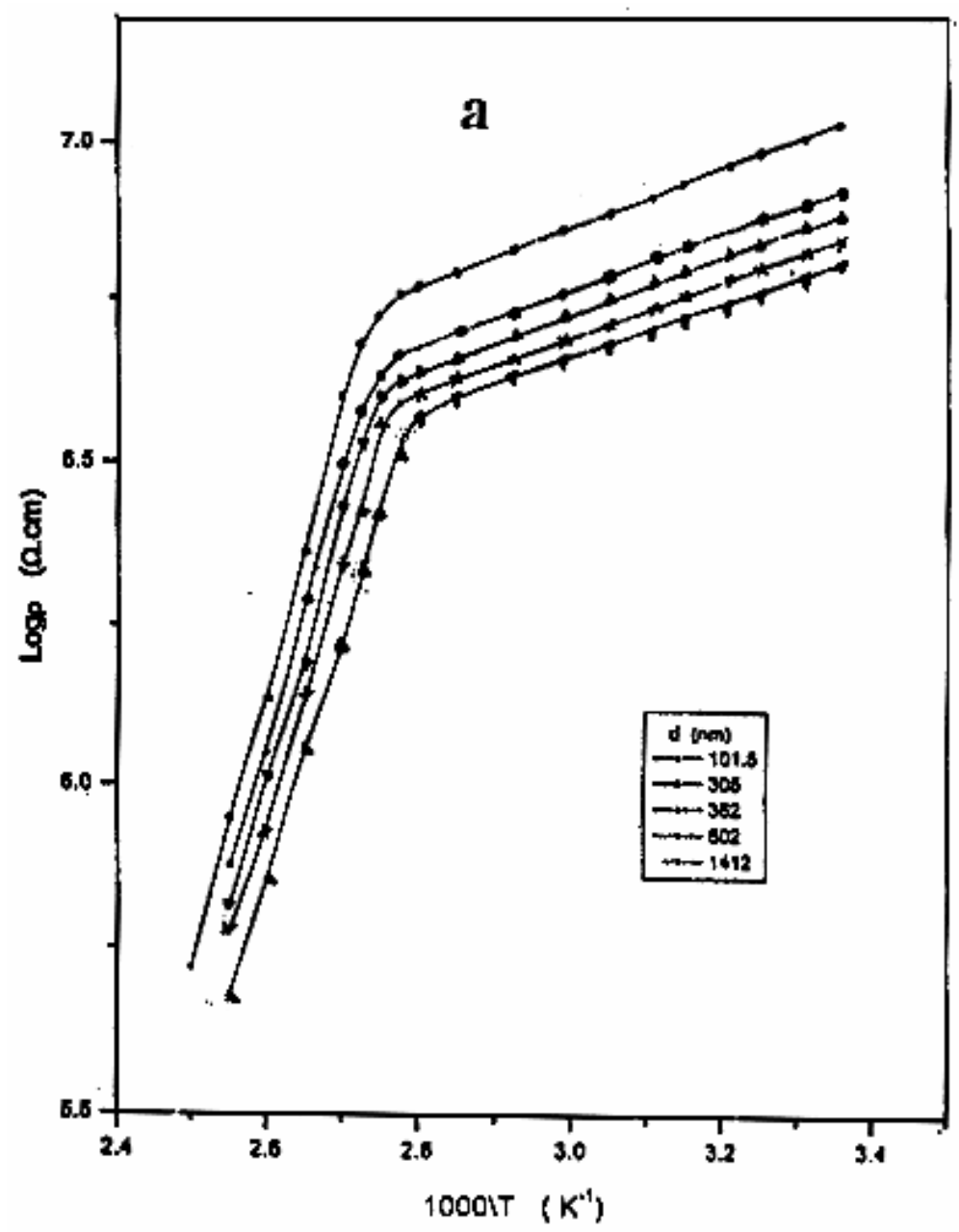

Fig .(4.a): The variation of dark electrical resistivity $p$ against film Thickness,d for CuPc thin films.

The dark electrical resistivity of $\mathrm{CuPc}$ thin films has been carried out in the temperature range $300-450 \mathrm{~K}$. The temperature dependence of resistivity is expressed as follows:

$$
\rho=\rho_{o} \exp \left(\Delta E / k_{B} T\right)
$$


where $\Delta \mathrm{E}$ is the thermal activation energy of the free charge carriers and $\mathrm{k}$ is the Bolzmann's constant. A graph of $\log \rho$ versus $1 / \mathrm{T}$ for $\mathrm{CuPc}$ films in thickness range 101-1415 $\mathrm{nm}$ is depicted in Fig. (4.b). From this figure, there are two linear regions and the corresponding activation energies $\Delta \mathrm{E}_{1}$ and $\Delta \mathrm{E}_{2}$ are thus obtained at $\mathrm{T}>380 \mathrm{~K}$ and $\mathrm{T}<380 \mathrm{~K}$, respectively. The thermal activation energy $\Delta \mathrm{E}_{1}$ is associated with an intrinsic generation process and $\Delta \mathrm{E}_{2}$ is associated with an impurity conduction. The change in the slope and hence the activation energy is interpreted as a change from extrinsic to intrinsic conduction [26].The mean values of $\Delta \mathrm{E}_{1}$ and $\Delta \mathrm{E}_{2}$ are 0.803 and $0.088 \mathrm{eV}$, respectively. Thus, in the intrinsic region the value of the thermal energy gap, $\mathrm{Eg}=3.1 \pm 0.02 \mathrm{eV}$, which is in agreement with the estimated band gap from the onset of the absorption spectrum of $\mathrm{CuPc}[27]$.

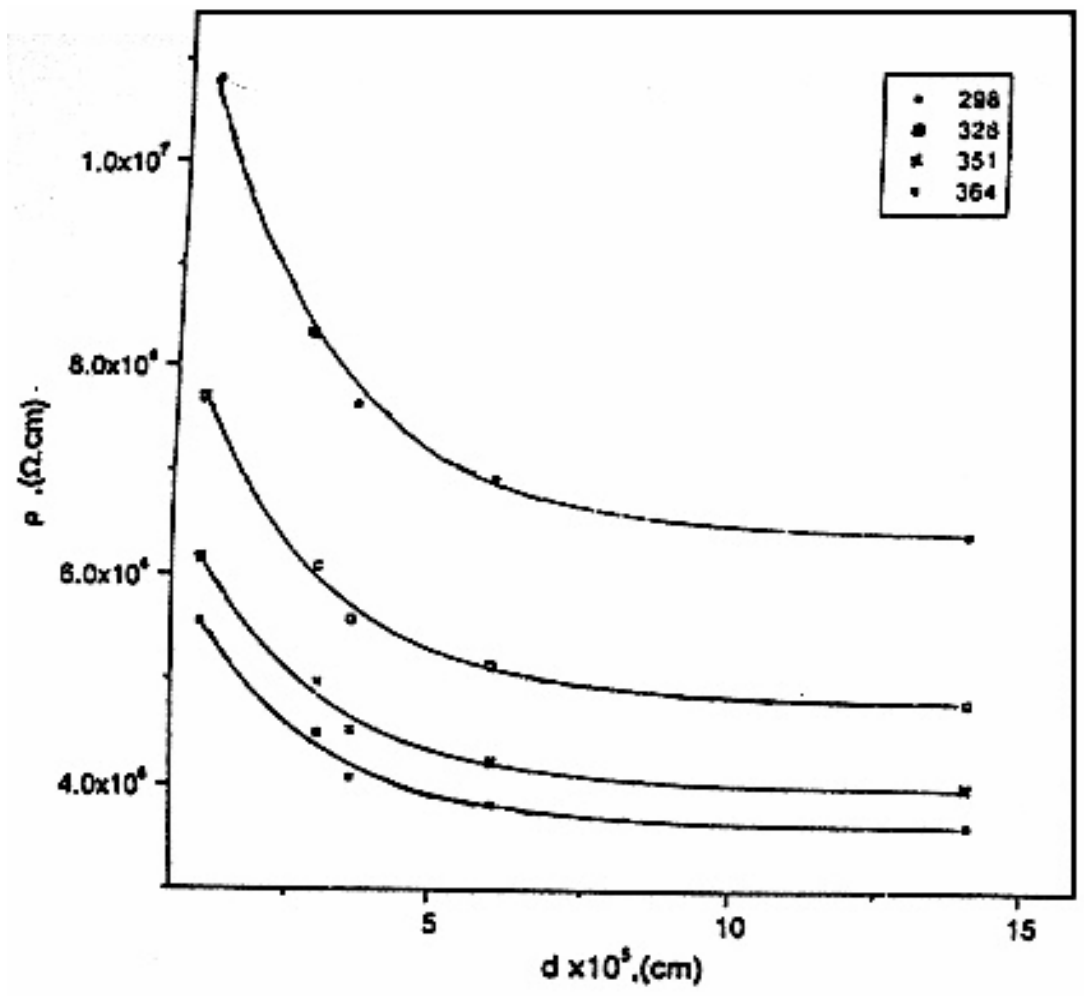

Fig (4.b) : The variation of dark electrical resistivity $p$ against temperature $\mathrm{T}$ of $\mathrm{CuPc}$ thin films.

In order to detect the type of conduction in CuPc thin film,the Seebeck coefficient was measured at different temperatures. Fig. (5) depicts the variation of Seebeck coefficients, S, versus temperature for different thicknesses of CuPc thin films. It can be noted that $\mathrm{S}$ is positive for the studied thicknesses all over 
the whole range of temperature. The same behaviour of $\mathrm{S}$ has been observed in $\mathrm{CuPc}, \mathrm{PbPc}$ and FePc [28,29,30]. A positive Seebeck coefficient can arise in several fashions; e.g. in an intrinsic semiconductor in which the mobility of the holes is greater than that of the electrons [31].



Fig .(5): The variation of Seebeck coefficient against temperature $\mathrm{T}$ for $\mathrm{CuPc}$ thin films.

\subsection{Space-charge-limited current investigations:}

\subsection{1 . Current density-voltage measurements:}

Figure (6) shows the dependence of current density $\mathrm{J}$ on voltage $\mathrm{V}$ for various samples in the thickness range 101-1415 $\mathrm{nm}$. For each characteristic there are two distinct voltage regions. At lower voltages, the slopes of the curves are approximately equal to unity, while at higher voltage levels, above a fairly well defined transition voltage $\mathrm{V}_{\mathrm{t}}$, the slopes are approximately equal to 3.6. In the lower voltage region, where $\mathrm{J}$ is proportional to $\mathrm{V}$, it may be described simply by Ohm's law for an p- type material, given by Lampert [32] as

$$
J=e p_{o} \mu_{h}\left(\frac{V}{d}\right)
$$

where $\mu_{\mathrm{h}}$ is the hole mobility and $\mathrm{p}_{\mathrm{o}}$ is the concentration of thermally activated holes in the valence band. The power -law conductivity above $V_{t}$ is typical of 
SCLC dominated by an exponential distribution of trap levels [33,34]. According to Lambert, the SCLC characteristics for a p-type semiconductor with an exponential distribution of trapping levels is given by [33,34]

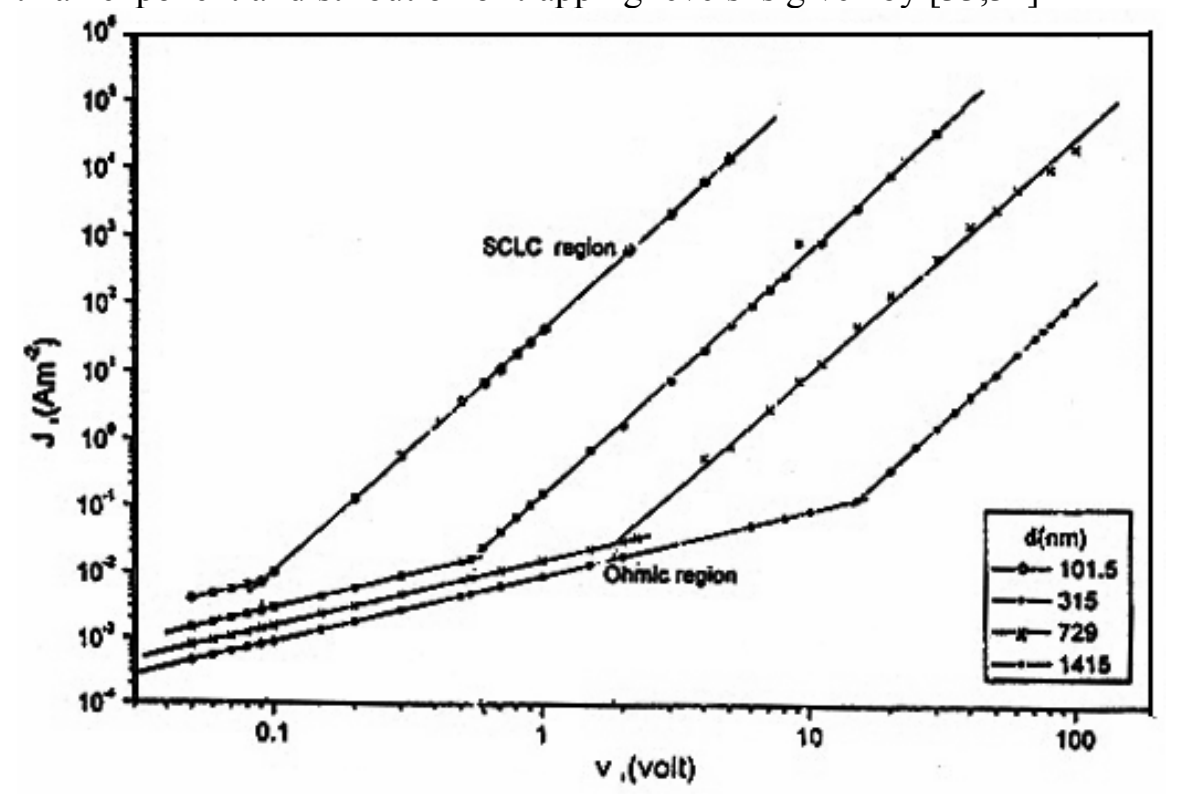

Fig (6): Dependence of current density J on applied voltage V for CuPc thin films.

$$
J=e \mu_{h} N_{V}\left(\frac{\varepsilon}{e P_{o} k_{B} T_{t}}\right)^{l} \frac{V^{l+1}}{d^{2 l+1}}
$$

where $\mathrm{N}_{V}$ is the effective density of states in the valence band edge, $\mathrm{P}_{\mathrm{o}}$ is the trap density per unit energy range at the valence band edge, and $\mu$ the hole mobility. The constant $l$ is the ratio $\mathrm{T}_{t} / \mathrm{T}$, where $\mathrm{T}$ is the room ambient temperature and $T_{t}$ is the temperature parameter describing the exponential trapping distribution $\mathrm{P}_{(\mathrm{E})}$ given as [33,34] .

$$
P(E)=P_{o} \exp \left(\frac{-E}{k_{B} T_{t}}\right) .
$$

where $\mathrm{P}_{(\mathrm{E})}$ is the trap density per unit energy range at an energy $\mathrm{E}$ above the valence band edge .

The total density of traps $\mathrm{N}_{\mathrm{t}}$,comprising the distribution, is given by evaluating $\int P(E) d E$ from the valence band edge to infinity $[33,34]$.

$$
N_{t}=P_{o} k_{B} T_{t}
$$


An analysis of the data below $\mathrm{V}_{\mathrm{t}}$ in Fig. 6 was performed using the mean value of $\mu_{\mathrm{h}}=\mathbf{5} \times \mathbf{1 0}^{-\mathbf{8}} \mathrm{m}^{2} \mathrm{v}^{-1} \mathrm{~s}^{-1}$ obtained hereafter in section (3.3.2) . From Equation (3) a mean value of $p_{o}=\mathbf{4} \times \mathbf{1 0}^{\mathbf{1 8}} \mathrm{m}^{-3}$ was derived. Our results fairly agree with previousely reported values $[26,35,36]$.

Above $\mathrm{V}_{\mathrm{t}}$ the power - law exponent $\mathrm{m} \approx 3.7$ implies that $l=2.7$ and $\mathrm{T}_{\mathrm{t}} \approx 810 \mathrm{~K}$ for a measuring temperature of $300 \mathrm{~K}$. These values are similar to those of $\mathrm{m} \approx 3.6, l=2.6$ and $\mathrm{T}_{\mathrm{t}} \approx 780 \mathrm{~K}$ derived for lead phthalocyanine [26]. Fig. (7) shows the thickness dependence of current density for SCLC region at certain fixed value of voltage at room temperature. Each point corresponds to different sample. The relatively small scatter of points about this line indicates that the concentration of holes is relatively constant from sample to sample [26], the slope of $\approx-6.5$ obtained in the SCLC region verifies that the space charge- limited conduction is dominated by an exponential distribution of traps. From Equation. (4) the slope in this region should be - $(2 l+1)$ implying that, $l \approx 2.6$, which coincides with the values deduced from the J-V characteristics, Fig. (6) The analysis of these results may be made also by adopting the value of $N_{t}=5 \times 10^{24} \mathrm{~m}^{-3}$, derived from the temperature measurements of section (3.3.2). This yields a value of $P_{o}=2 \times 10^{43} \mathrm{~J}^{-1} \mathrm{~m}^{-3}$ using Equation (6), which together with the value of $\mathrm{T}_{\mathrm{t}}(810 \mathrm{~K})$ given above fully characteristize the trap distribution of Equation.(5). The values of $\mathrm{N}_{\mathrm{t}}$ and $\mathrm{P}_{\mathrm{o}}$ are in the same order with the values deduced by other workers $[33,34]$.

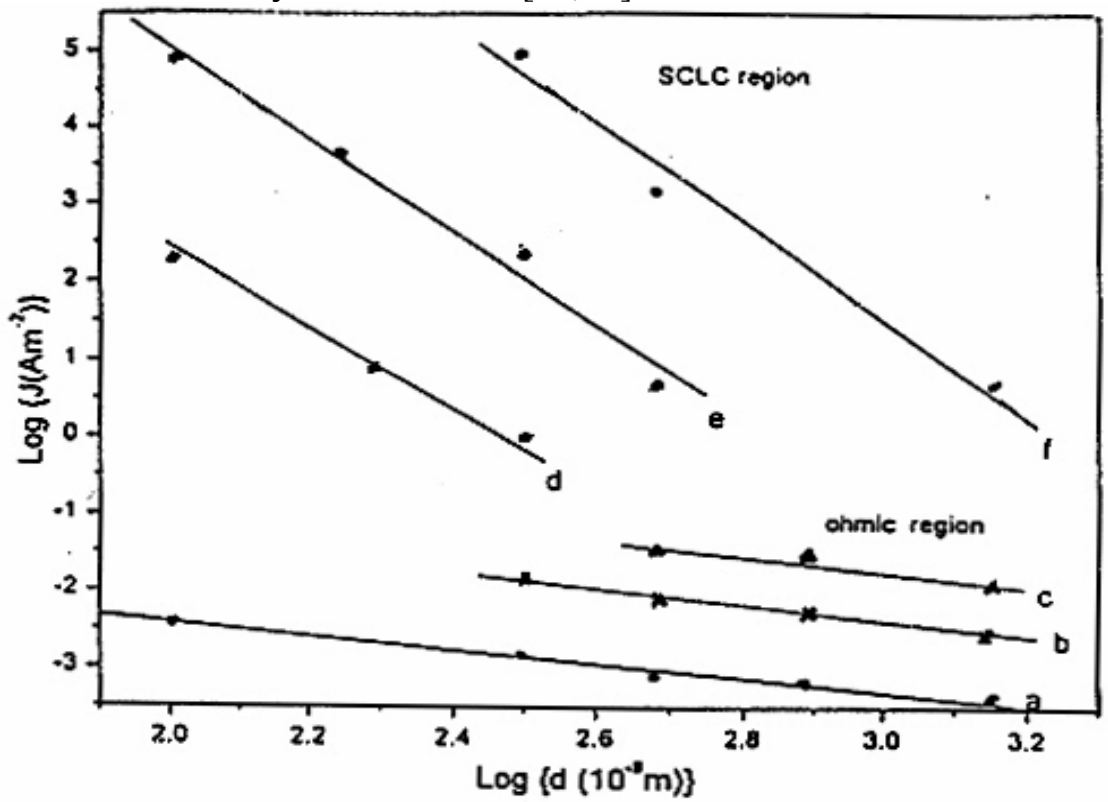

Fig .(7): Dependence of current density J on film thickness. 


\subsubsection{Current density - temperature measurements:}

Current density tsemperature measurements allow the determination of additional information concerning the film material. Fig. (8) shows temperature dependence of the current density, $\mathrm{J}$ in the temperature range $310-450 \mathrm{~K}$ for a sample of thickness $729 \mathrm{~nm}$ at various fixed voltages in the SCLC region. On the basis of Equations (4), (5) and (6), it was shown that the plots of $\log \mathrm{J}$ versus $\frac{1}{T}$ at constant voltage in the SCLC region gives

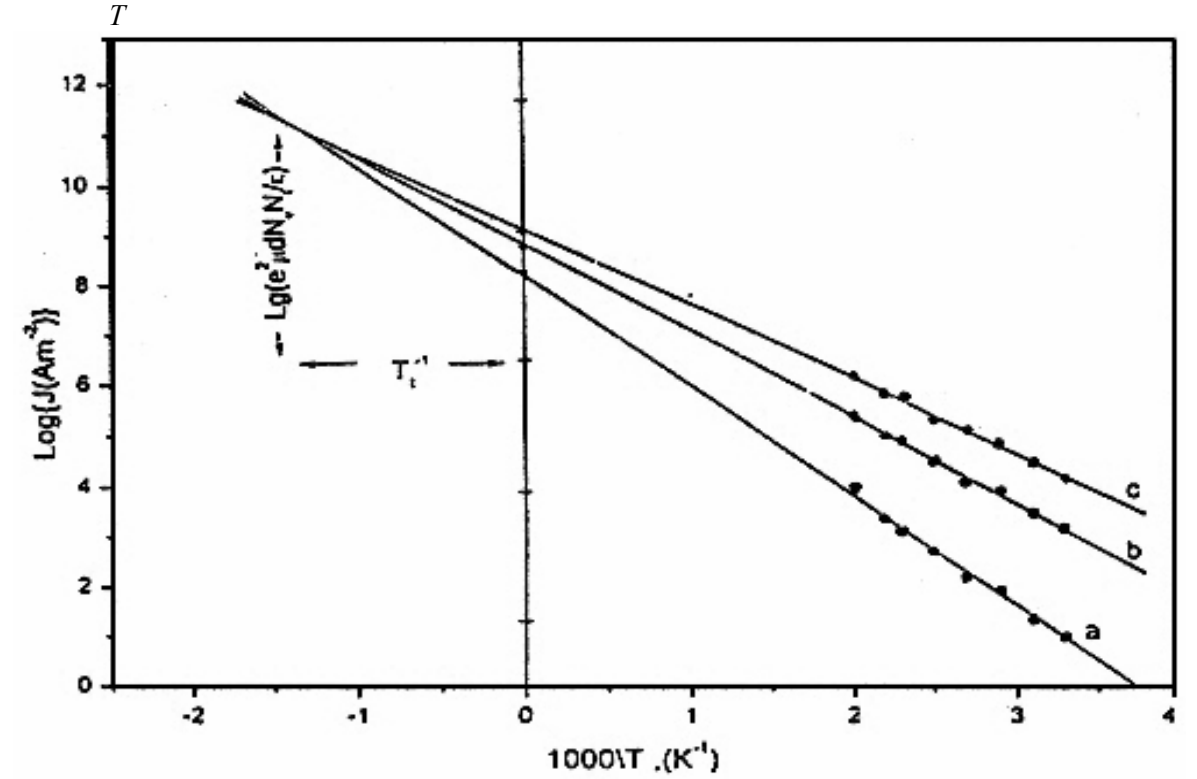

Fig .(8): Dependence of current density $\mathrm{J}$ on reciprocal temperature as a function of applied voltage for CuPc thin films of thickness $729 \mathrm{~nm}$.

$$
\text { slope }=\frac{d(\log J)}{d\left(\frac{1}{T}\right)}=T_{t} \log \left(\frac{\varepsilon V}{e d^{2} N_{t}}\right)
$$

From which, the values of $\mathrm{N}_{\mathrm{t}}$ can be obtained .

The intercept on the $\log \mathrm{J}$ axis is given by

$$
\log J_{o}=\log \left(\frac{e \mu_{h} N_{V} V}{d}\right)
$$

Using the value of $\mathrm{N}_{\mathrm{v}}=10^{27} \mathrm{~m}^{-3}[33,34]$ for metal phthalocyanine derivatives, Equation (8) yields mobilities between $(4-6) \times 10^{-8} \mathrm{~m}^{2} \mathrm{v}^{-1} \mathrm{~s}^{-1}$. On the extrapolation, it is predicted that all $\log \mathrm{J}$ versus $1 / \mathrm{T}$ plots should intersect at a common point, whose coordinates are given by $[35,36]$. 


$$
\log J=\log \left(\frac{e^{2} \mu_{h} d N_{V} N_{t}}{\varepsilon}\right) \text { and } \frac{1}{T}=-\frac{1}{T_{t}}
$$

These coordinates are independent of the applied bias voltages .

$\mathrm{J}_{\mathrm{o}}$ represents the current density at infinite temperature $(1 / \mathrm{T}=0)$. Assuming the value of $\mu_{\mathrm{h}}$ derived from Equation.(9), a value of $\mathrm{N}_{\mathrm{t}}$ may therefore be obtained using the coordinates of the intersection points in Fig. (8). The measured values of $\mathrm{N}_{\mathrm{t}}$ lie in the range $(5-7) \times 10^{24} \mathrm{~m}^{-3}$

The exponential trap distribution of Equation.(5) associated with SCLC is thus completely specified and is shown in Fig. (9) for $\mathrm{CuPc}$ thin films of thickness $729 \mathrm{~nm}$.

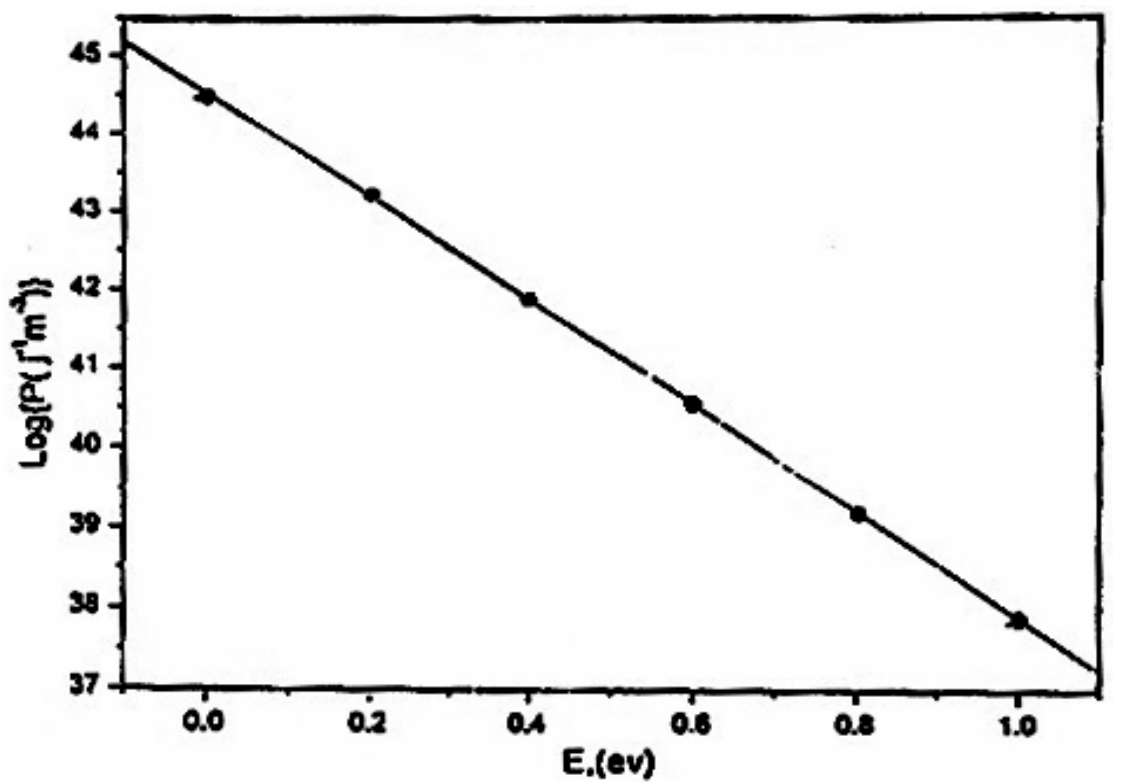

Fig .(9): Derived trapping distribution (exponentially) as a function of energy above the valence band edge for CuPc of thickness $729 \mathrm{~nm}$.

\section{Conclusions:}

X-ray diffraction patterns obtained for powder and thin films of thermally evaporated copper phthalocyanine show that the stable monoclinic $\beta$-phase exist in both specimens. The crystallite size of $\mathrm{CuPc}$ thin films increases with increasing either the film thickness or the annealing temperature. The dark electrical resistivity measured for $\mathrm{CuPc}$ thin films decreases with increasing the film thickness. Two activation energies are obtained for temperature ranges above and below $380 \mathrm{~K}$ pointing to a change from extrinsic to intrinsic 
conduction. Seebeck coefficient measurements showed that $\mathrm{CuPc}$ thin films behave as p-type semiconductor. The positive Seebeck coefficint in an intrinsic semiconductor is attributed to the difference in the free charge carrier mobilities. Measurements of current density as a function of applied voltage revealed that conductivity was ohmic at low voltages and space-charge-limited at higher voltages. The latter is due to the assistance of exponential of trap levels above the valence band. Measurements of current density as a function of temperature allowed mean values of the mobility and trap concentration to be estimated from the higher temperature SCLC data.

\section{References:}

1. R.D.Gould and R.I. Blyth , phys. stat. sol. (a), 120, K57 (1990).

2. J.M. Assour and W.K. Kahn, J.Amer. Chem. Soc., 87, 207 (1965).

3. G.Susich, Anal.Chem., 22, 425 (1950).

4. F.R. Tarantino, D.H. Stubbs, T.F. Cooke, and L.A. Melsheimer, Am. Ink Maker, 29, 425 (1950).

5. A.A. Ebert, Jr., and H.B. Gottlieb, J. Amer. Chem. Soc., 74, 3806 (1952).

6. F.W.Karasek and J.C.Decius, ibid., 74, 4716 (1952).

7. M.Shigemitsu, Bull. Chem.Soc., Jap.,32, 607 (1959).

8. J.W.Eastes, U.S.Patent, 2, 620 (1956).

9. J.F.Byrne and P.F.Kurz, U.S.Patent, 3, 989 (1967).

10. A.N. Sidorov and I.P.Kotlyar, Sov. Phys. Opt. and Spectrosc., 11, 92 (1961).

11. M.A.M.Seyam and A.Elfalaky, Vacuum, 57, 31 (2000).

12. R.P . Linstead and Z.M.Robertson, J. Chem. Soc., 1195 ( 1936 ).

13. A.K. Hassan and Gould, phys. stat. sol.(a), 132, 91 (1992)

14. S.F. Shihub and R.D. Gould, phys. stat. sol.(a), 139 (1993).

15. R.A. Collins and K. A. mohamed, J. Phys. D: Appl. Phys., 21, 154 (1988).

16. F.W. Kutzler, W.R. Barger, A.W. Snow and H. Wohltjen, Thin Solid Films 155, 1 (1987).

17. A.Szczurek and K.Lorantz, Mat.Sci., 9, 114 (1983).

18. A.K.Abass, A.Krier, and R.A Collins, phys. stat.. sol. (a), 142, 435 (1994).

19. A.K.Hassan, R.D.Gould. Int.J. Elect., 74, 5 (1993).

20. A. Wilson and R.A. Collins, Sensors and Actuators, 12, 389 (1987).

21. M.A.M.Seyam and A.Elfalaky, Vacuum, 57, 31 (2000).

22. James H.Sharp and Marcel Lardon, J.Phys. Chem., 72, 3230 (1968).

23. A.K. Hassan and Gould, phys. stat. sol.(a), 132, 91 (1992).

24. C.RTellier, Thin Solid Films, 51, 311 (1978).

25. A.K.Hassan, R.D.Gould and A.K.Ray, phys. stat. sol. (a), 158, K23 (1996).

26. A.Ahmed and R.A Collins, phys. stat. sol. (a), 123, 201 (1991). 
27 M.M.El-Nahass, F.S. Bahbri and S.R.Al-Harbi, Egypt. J. Sol., 24, 11 (2001)

28. A.Belghachi and R.A.Collins, J.Phys., D:Appl. Phys., 21, 1647 (1988).

29. J.J. Broply and J.W Buttrey, "Organic Semiconductors", New York, p149 (1962).

30. H. Soliman, M.M. El-Nahass, A.M.farid, A.A. M. Farag, and A.A. El-Shazly, to be published.

31. T.G. Abdel Malik, A.M. Abdeen, H.M..El-Labany and A.A. Aly, phys. stat. sol. (a), 72, 99 (1982).

32. M.A.Lampert, Rep. Progr. Phys., 27, 329 (1964).

33. A.Ahmed and R.A Collins, phys. stat. sol. (a), 123, 201 (1991).

34. R.A.Collins and K.A.Mohamad, Thin Solid Films, 145, 133 (1986).

35. R.D. Gould, Thin Solid Films, 125, 63 (1985).

36. R.D. Gould, J. Phys. D.: Appl. Phys. 9, 1785 (1986). 